\title{
Polyarteriitis nodosa with real arteries and vein involvement and uroligical manifestations: case
} report

\begin{abstract}
Current Nomenclature defines polyarteriitis nodosa (PAN) as a medium vessels vasculitis a necrotizing arteritis of medium or small arteries without glomerulonephritis or vasculitis in arterioles, capillaries, or venules; and not associated with ANCA. PAN typically affects skin, joints, peripheral nervous system, digestive tract and kidneys. Here we present a case of PAN with extremely severe combined left kidney damage: renal artery aneurism and occlusion with peri-vasal hematoma, multiple kidney infarctions, renal vein thrombosis, necrotizing ureteritis with periureteritis, with left orchitis and with skin and joints involvement, successfully treated by nephrectomy and immunosuppression.
\end{abstract}

Keywords: necrotizing vasculitis, renal artery aneurism, kidney infarction, renal vein thrombosis, ureteritis, orchitis
Volume 5 Issue 3 - 2017

\author{
Elena V Zakharova, ${ }^{1,2}$ Andrey Shubin, ${ }^{3}$ Tatyana \\ Makarova,' Olga Vorobyova ${ }^{4}$ \\ 'Department of Nephrology, City Hospital n.a. S.P. Botkin, Russia \\ ${ }^{2}$ Department of Nephrology and Hemodialysis, Russian Medical \\ Academy of Postgraduate Education, Russia \\ ${ }^{3}$ Department of Vascular Surgery, City Hospital n.a. S.P. Botkin, \\ Russia \\ ${ }^{4}$ Departmen of Pathology, National Centre of Clinical \\ Morphology, Russia
}

Correspondence: Elena V Zakharova, City Clinical Hospital
n.a. S.P. Botkin, I I 5284, 2-nd Botkinsky proezd, 5, Moscow,
Russia,Tel 7499728 829।, Email helena.zakharova@gmail.com

Received: July 31, 2017 | Published: September 21, 2017

\begin{abstract}
Abbreviations: ACCP, anti-cyclic citrullinated peptide antibodies; ACTH, adrenocorticotropic Hormone; ACR, american college ofrheumatology; AFP, alpha-fetoprotein;ANCA, antineutrophil cytoplasm antibodies; BP, blood pressure; CRP, c-reactive protein; CT, computer tomography; DNA, deoxyribonucleic Acid; EBV, epsteinbarr Virus; ECG, electrocardiogram; ECHO-CG, echocardiography; ESR, erythrocytes sedimentation rate; FFS, five factor score; FSH, follicle-stimulating hormone; $\mathrm{Hb}$, Hemoglobin; HBsAg, hepatitis B serum antigen; HCG, human chorionic gonadotropin; HCV, hepatitis C Virus; H\&E, haematoxylin-eosin; HEENT, Head, eyes, ears, nose, Throat; HIV, human immunodeficiency Virus; hpf, high power field; i.v., intravenous; LH, luteinizing hormone; PAS, periodic acidshiff; Plt, platelets; PSA, prostate-specific antigen; RF, rheumatoid factor; ROM, range of motion; rpr-test, rapid plasma regain test; RR, respiratory rate; SG, specific gravity; TBC, total blood count; VCA, viral capsid antigen; WBC, white blood cells
\end{abstract}

\section{Introduction}

Current Nomenclature defines polyarteriitis nodosa (PAN) as a medium vessels vasculitis - a necrotizing arteritis of medium or small arteries without glomerulonephritis or vasculitis in arterioles, capillaries, or venules; and not associated with ANCA. ${ }^{1}$ PAN typically affects skin, joints, peripheral nervous system, digestive tract and kidneys. ${ }^{2}$ Here we present a case of PAN with extremely severe combined kidney damage.

\section{Case presentation}

28 years old Caucasian man, admitted May 312017.

\section{Main complains}

General weakness, fever, back pain, muscle pain, weight loss.

\section{Previous medical history}

Unremarkable.

\section{History of current disease}

In April 2016 he developed painful skin nodules on his upper and lower extremities, and scrotum discomfort. Four months later he got intermittent fever $38^{\circ} \mathrm{C}$, muscle and joints pain, and started taking NSAID's with temporary effect. Outpatient work-up found arterial hypertension (BP 140/90-180/100 mm Hg) and elevated ESR; TBC, blood chemistry and urinalysis were normal; kidney and abdomen ultrasound and ECHO-CG were unremarkable. He was diagnosed with erythema nodosum and continued on NSAID's. His symptoms persisted; he lost 5kilos of weight. Within next three months work-up found proteinuria $0.1 \mathrm{~g} / \mathrm{L}$ and microhematuria $4-5 \mathrm{RBC} / \mathrm{hpf}$, Plt count 448x109/L, CRP $70 \mathrm{mg} / \mathrm{L}$, and IgG antibodies against EBV and VCAEBV. PCR test for EBV DNA was negative; RF, ACCP, anti-DNA, antinuclear and antiphospholipid antibodies, pANCA, cANCA - all within normal range; blood culture was negative. He was diagnosed with EBV chronic infection and started on Valacyclovir and Isoprinosine without any improvement of his signs and symptoms.

After 4months of antiviral treatment his CRP was now elevated up to $98 \mathrm{mg} / \mathrm{L}$, he developed mild leukocytosis (WBC 10.4x109/L), his thrombocytosis persisted, and his serum creatinine raised to $150 \mu \mathrm{mol} / \mathrm{L}$. Ultrasound now found left kidney shrinkage with renal blood flow reduction and left epididymis enlargement; right kidney and right epididymis were otherwise normal. He was suspected with left testicle neoplasm; however his LH, FSH, estradiol, progesterone, prolactin, testosterone, ACTH, cortisol, aldosterone, AFP, PSA and free beta HGC were all normal. Antiviral treatment was discontinued, and he underwent contrast-enhanced abdomen CT and CT-angiography, which showed pseudo-aneurism of the left artery, left artery proximal occlusion and reduced left kidney excretory function (Figures 1 \& 2). Eleven months after his first presentation he was referred to our hospital and admitted to the vascular surgery.

\section{At admission}

Alert, oriented. Body temperature $37.8^{\circ} \mathrm{C}$, RR 19 per minute, pulse regular 92 per minute, BP 160/95 mm Hg. Undernourished. Skin 
pale, moist, with few slightly painful nodular erythematous eruptions on the arms and shoulders. No peripheral oedema. HEENT and neck otherwise normal. No palpable peripheral lymph nodes. Joints: no swelling, ROM normal. Lungs: clear. Heart: rhythm regular, no murmurs. Abdomen soft, slightly tender in the upper left quadrant, bowel sounds normal. Liver $+2 \mathrm{~cm}$ below costal margin, non-painful, spleen and kidneys not felt. Urine output $850 \mathrm{~mL} /$ day, urine normally coloured.

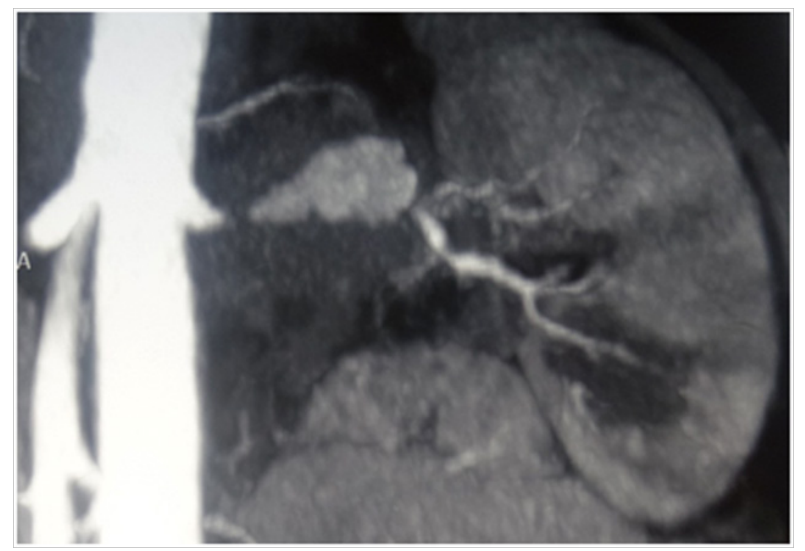

Figure I CT scan, showing left renal artery pseudo-aneurism and proximal occlusion.

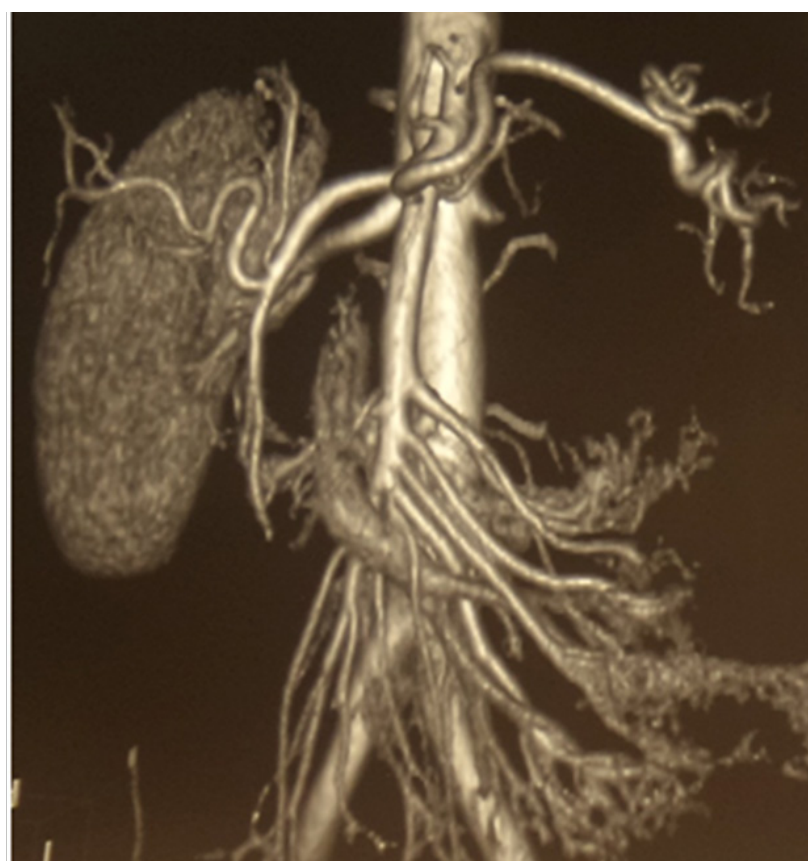

Figure 2 CT scan, showing blood flow reduction of the left kidney.

\section{Work up}

TBC: Hb 7.1 g/L, WBC 14.5x109/L, Plt 612x109/L, ESR 52mm/h.

Blood chemistry: Creatinine $163 \mu \mathrm{mol} / \mathrm{L}$, urea $13.1 \mathrm{mmol} / \mathrm{L}$, uric acid $378 \mu \mathrm{mol} / \mathrm{L}$, total protein $81 \mathrm{~g} / \mathrm{L}$, serum iron $10.7 \mu \mathrm{mol} / \mathrm{L}$; CRP $165 \mathrm{mg} / \mathrm{L}$; electrolytes, glucose, albumin, cholesterol, bilirubin, liver enzymes - within normal range.

Urinalysis: SG 1020, protein $1.0 \mathrm{~g} / \mathrm{L}, \mathrm{RBC} 3-4$, WBC 75-80 hpf.

Infections screening: RPR-test for Treponema Pallidum, HBsAg, anti-HCV and anti-HIV-antibodies negative, tuberculin skin test - all negative.
ECG; plain chest X-Ray and ECHO-CG: Unremarkable.

Abdomen and kidneys ultrasound: Liver slightly enlarged; pancreas and spleen otherwise normal. Right kidney 110x45 mm, with normal parenchyma $17 \mathrm{~mm}$. Left kidney $84 \times 50 \mathrm{~mm}$, with hyperechoic parenchyma $14 \mathrm{~mm}$. Space-occupying mass in the projection of the main stem of the left renal artery - most likely renal artery aneurism with aneurism thrombosis and paravasal hematoma. Pelvis not dilated.

Urologist consults: Left-side epididymitis and orchitis.

Neurologist consults: No signs or symptoms of any neurologic disease.

Diagnostic considerations and treatment: At that point left nephrectomy was considered and nephrologist was invited to see the patient. Given the combination of weight loss, orchitis, myalgia/ arthralgia, arterial hypertension, elevation of serum creatinine and renal artery aneurism, the patient was diagnosed with polyarteriitis nodosa. June 22017 nephrectomy was performed.

Excised kidney pathology: Light microscopy sections of formalin fixed paraffin embedded tissue were stained with H\&E, PAS and Masson's trichrome.

Renal artery: Figures $3 \& 4$ Fragment of artery ofelastic type, severely thickened due to irregular sclerosis and hyalinosis with impaired histoarchitectonics of the wall layers and integrity. Internal elastic membrane segmentally preserved. Severe subendothelial fibrosis with focal lympho-histiocytic infiltration and hemosyderophage clusters. Vessel lumen and internal surface of the vessel are covered with thrombotic masses with secondary changes, presented by organization, cell infiltration and recanalization (neo-formed vessels), and fresh thrombotic masses without cell reaction. Perivascular lymph node with prominent sclerosis, and lymphoid tissue hypoplasia presented by few lymphoid follicles with attenuated centers. Muscular type arteries changes are the same as in the renal tissue and ureter wall arteries.

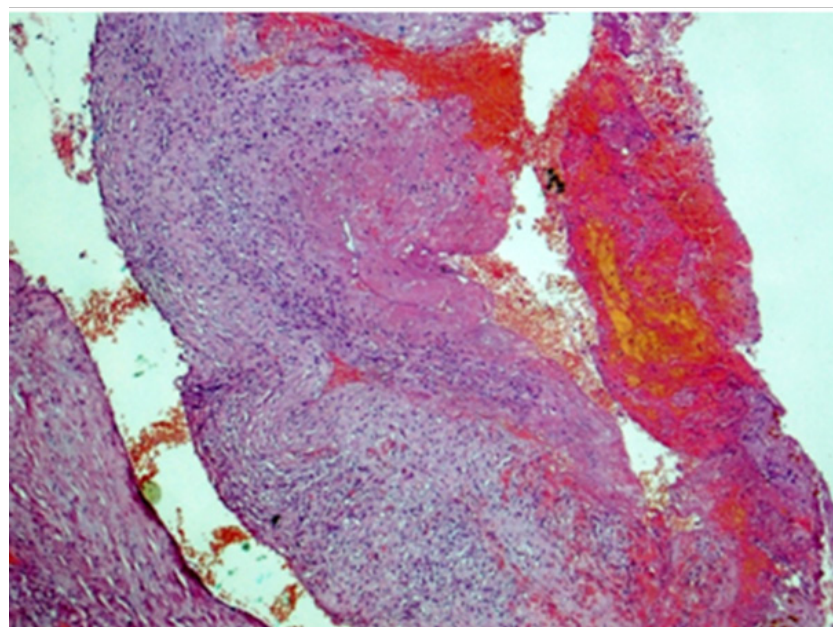

Figure 3 Renal artery wall is extremely thickened due to severe irregular sclerosis and hyalinosis, with disorganization of layers and broken wall integrity. In the lumen, there is fresh thrombotic masses connected to the wall and presented by fibrin, erythrocytes, and small clumps of hemosiderophages. Hematoxylin \& Eosin, $x 40$.

Kidney: Arterioles and small arteries walls otherwise normal. Middle arteries walls are diffuse thickened due to the fibrous connective tissue with impaired histoarchitectonics of the wall layers and subtotal limen obstruction. Large arteries walls are thickened due to edema, 
massive transmural and perivascular infiltration with neutrophils and histiocytes, and irregular fibrous connective tissue with impaired histoarchitectonics of the wall layers. Multiple pseudo-aneurisms in the zones of large arteries bifurcation with the severe edema and prominent walls infiltration. Multiple cortical macrofocal ischemic and hemorrhagic renal tissue necrosis. Preserved renal tissue presented by normal glomeruli. Total acute tubulonecrosis without tubular atrophy or interstitial fibrosis (Figures $5 \& 6$ ).

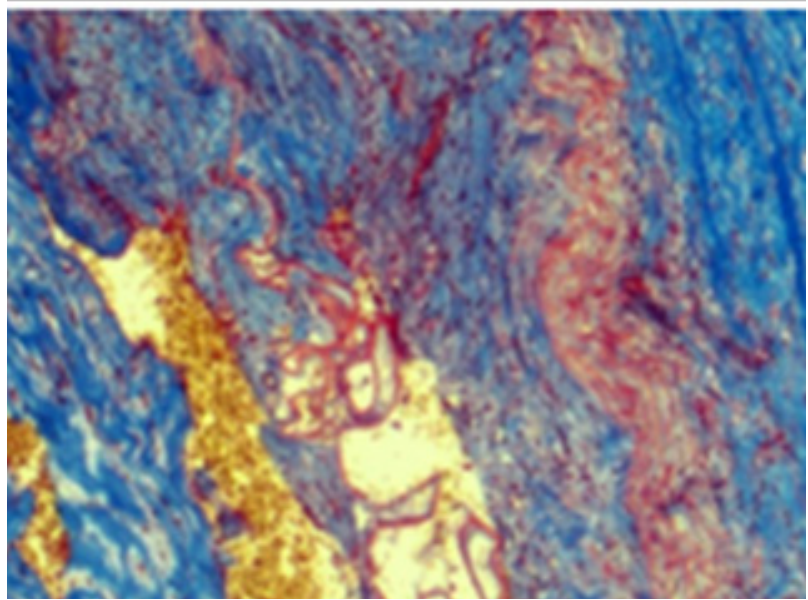

Figure 4 Renal artery wall shows the segmentally preserved internal elastic membrane, marked subendothelial fibrosis and severe irregular sclerosis and hyalinosis. Masson's trichrome; $\times 40$.

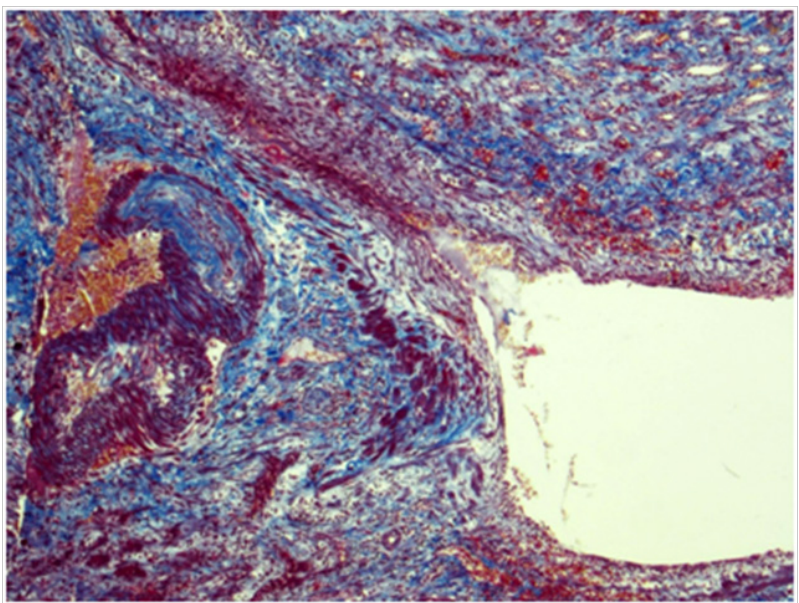

Figure 5 Kidney. The wall of a large caliber artery is markedly thickened, forms pseudoaneurismal widening and shows histological architecture disturbance due to the following: edema, dense transmural and perivascular infiltration presented by neutrophilic leukocytes and histiocytes, and irregular coarse fibrosis. Nearby the wall of a medium caliber artery demonstrates severe irregular focal fibrosis with total occlusion of its lumen. Masson's trichrome; $\times 40$.

Ureter: Erosive-ulcerouslesions of mucosa with massive fibrinoleucocyte excaudate. Ureter wall is thickened due to the severe edema and massive leucocyte infiltration of all layers; with multiple intramural necrosis and prominent neutrophil karyorhexis. Small, medium and large arteries walls are thickened due to the irregular neutrophil infiltration, with circular fibrinoid necrosis of small and medium arteries walls; and focal impaired histoarchitectonics and integrity of large arteries walls due to severe infiltration; with multiple pseudo-aneurism formation. Diffuse severe fibrous periureteritis with massive fibrin deposition and leucocyte infiltration with prominent karyorhexis (Figures 7-9).

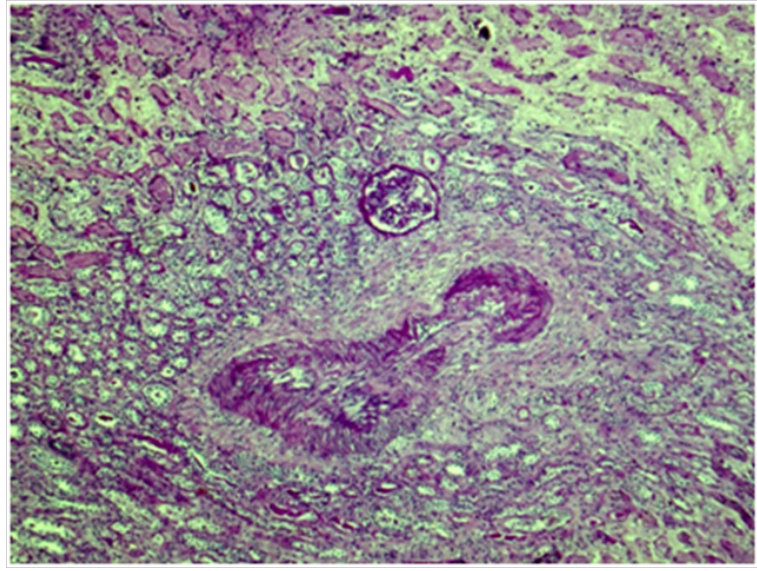

Figure 6 Kidney. The wall of a medium caliber artery shows histological architecture disturbance due to severe irregular fibrosis with marked luminal narrowing. The upper third of the picture demonstrates cortical necrosis with total ischemic tubular epithelial lesion; nearby the glomerulus is unremarkable. Periodic acid-Schiff, $x 40$.

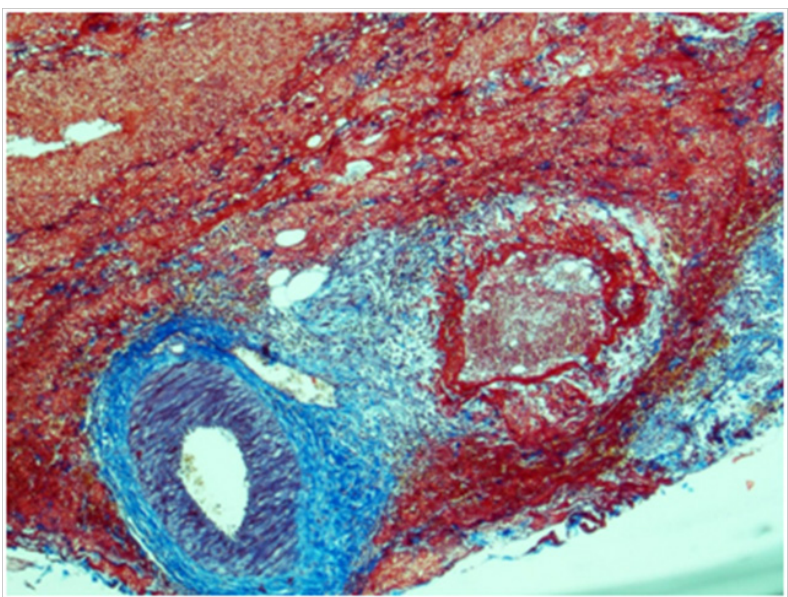

Figure 7 Ureter. The wall of a medium caliber artery is sharply thickened due to perivascular fibrosis, forms pseudoaneurismal widening with severe edema and overt circular fibrinoid necrosis. Masson's trichrome; $\times 40$.

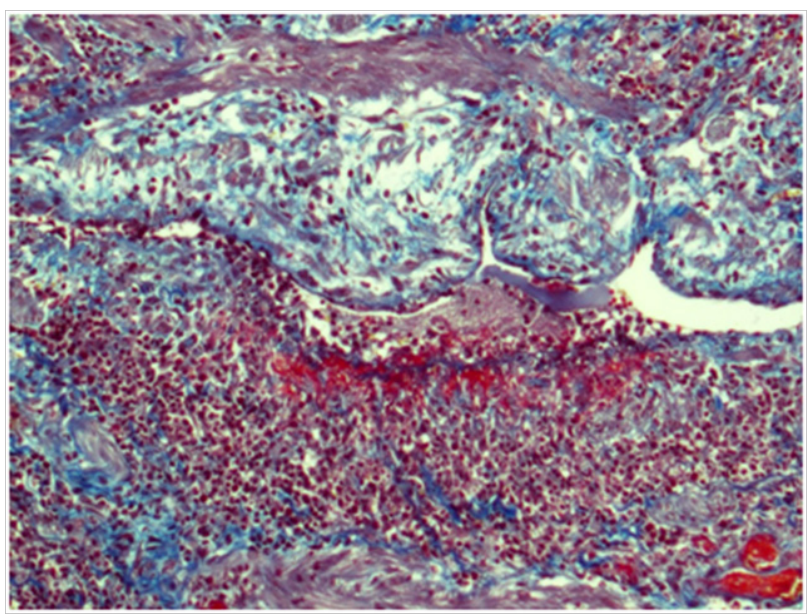

Figure 8 Ureter. The wall of a medium caliber artery is extremely thickened with subtotal luminal narrowing due to severe subendothelial edema and focally distributed dense neutrophilic infiltration with fibrinoid necrosis. Masson's trichrome; $\mathbf{x} 100$. 


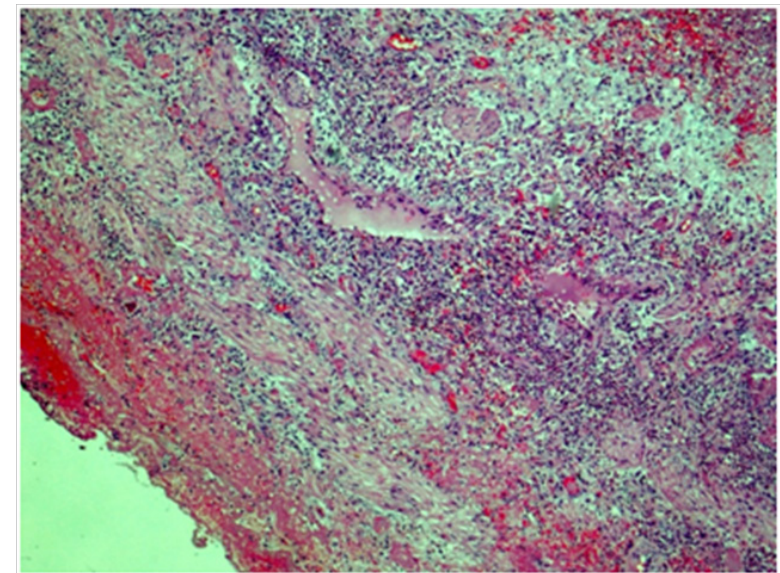

Figure 9 Ureter, outer part of the wall. Throughout the wall there is a histological architecture disturbance due to severe edema, massive transmural leukocytic infiltration, multiple intramural necrosis, and overt neutrophilic karyorrhexis. There is marked fibrinous periureteritis with fibrin masses and leukocytic infiltration. Hematoxylin \& Eosin, $x 40$.

Renal vein: With total obstruction of the lumen by the sludged thrombi.

Pathologists conclusion: Poliangiitis nodosa - diffuse segmental transmural necrotizing and sclerosing vasculitis with renal artery, small, medium and large intrarenal and ureter wall arteries, with pseudo-aneurism formation and thrombosis; macrofocal ischemic and hemorrhagic renal infarctions; renal vein thrombosis. Phlegmonousulcerous ureteritis fibrinous periureteritis.

Final diagnosis and further treatment: Pathology evaluation confirmed the diagnosis of poliarteriitis nodosa. Within 2 weeks after the surgery patient was moved to nephrology and started on oral prednisone $50 \mathrm{mg} /$ day and cyclophosphamide i.v. "pulses" $500 \mathrm{mg}$ every 2 weeks.

Treatment results: At the last evaluation after 6weeks of treatment patient is doing well. His body temperature is normal, BP is $130 / 80$ $\mathrm{mm} \mathrm{Hg}$, his skin lesions resolved, and he gained 2kilos of weight. Work-up showed Hb 13.7g/dL, WBC 10.5x109/L, Plt 264x109/L, ESR $17 \mathrm{~mm} /$ hour, CRP $1.0 \mathrm{mg} / \mathrm{L}$, serum creatinine $109 \mu \mathrm{mol} / \mathrm{L}$, his urinalysis are normal (proteinuria and microhematuria not detected). He already received $1500 \mathrm{mg}$ of cyclophosphamide and continued on cyclophosphamide "pulses" $500 \mathrm{mg}$ every other week and oral prednisone $45 \mathrm{mg}$ /day with slow tapering to $30 \mathrm{mg}$ /day.

\section{Discussion}

Our patient presented with fever, erythema nodosum, muscle and joint pain, orchitis, weight loss, elevated ESR and CRP, thrombocytosis, leukocytosis, anemia, proteinuria, arterial hypertension, impaired kidney function, renal artery pseudo-aneurism and occlusion, and peri-renal mass. Matching clinical presentation over ACR 1990 criteria. ${ }^{3}$ he had 6 out of 10 most characteristic disease features:
i. Weight loss of $4 \mathrm{~kg}$ or more - present
ii. Livedo reticularis -absent
iii. Testicular pain/tenderness - present
iv. Myalgia or leg weakness/tenderness - present
v. Mononeuropathy or polyneuropathy - absent

vi. Diastolic blood pressure greater than $90 \mathrm{~mm} / \mathrm{Hg}$ - present

vii. Elevated blood urea nitrogen or creatinine level unrelated to dehydration or obstruction - present

viii. Presence of hepatitis B surface antigen or antibody in serum -absent

ix. Arteriogram demonstrating aneurysms or occlusions of the visceral arteries - present

x. Biopsy of small- or medium-sized artery containing polymorphonuclear neutrophils - not evaluated at the time of diagnosis

According to the ACR criteria, the presence of at least 3 of these features is required for classification of disease as PAN with $82 \%$ sensitivity and $87 \%$ specificity rate; therefore, the diagnosis of PAN in our patient was certain. In addition, fever, skin nodules, arthralgia's, elevated ESR and CRP, thrombocytosis, leukocytosis and moderate proteinuria, which our patient demonstrated, are quite characteristic, even though non-specific features of PAN. ${ }^{4}$ Moreover, male gender also matching with typical for PAN male-to-female ratio, which is 1.6-2: $1 .^{5}$ The absence of hepatitis $\mathrm{B}$, which is known to be strongly related with PAN, does not exclude the diagnosis. It was estimated that hepatitis B is causative for $30 \%$ and more recently - for $7-10 \%$ of PAN cases. ${ }^{6,7}$ Given that, he met ACR criteria for PAN even before the kidney pathology became available.

Renal manifestations in our patient were quite severe. Proteinuria and serum creatinine $>1.58 \mathrm{mg} / \mathrm{dL}(139.7 \mu \mathrm{mol} / \mathrm{L})$, which both he demonstrated at presentation, are 2 out of 5 negative prognostic factors for PAN; presence of 2 and more factors is related to $45.9 \%$ 5 -years mortality according to the prognostic Five Factor Score (FFS). ${ }^{8}$ Aneurismal ruptures are one of the most serious complications of PAN, and may occur in many involved organs, including kidneys; in such cases aneurismal ruptures are causative for peri-renal hemorrhage. The typical clinical presentation is a loin pain, significant $\mathrm{Hb}$ fall, hypotension and a peri-renal mass, which was also found in our patient. In some cases surgical evacuation of the hematoma or partial/complete nephrectomy is necessary. ${ }^{9-11}$

In general, histology in PAN reveals a focal necrotizing arteritis of mixed cellular infiltrate within the vessel wall. Necrotizing arteritis begins as a segmental necrotizing inflammation of arteries with conspicuous infiltration of neutrophils and monocytes. This typically induces fibrinoid necrosis characterized by accumulation of plasma proteins in injured tissue, including coagulation factors that are converted to fibrin. ${ }^{1}$. Kidney pathology data for PAN are scarce; kidney biopsy is performed only in exceptional cases due to the risk of aneurysmal rupture and bleeding. ${ }^{12}$ However, there are few reports, confirming renal involvement with typical necrotizing vasculitis in patients with PAN. ${ }^{13,14}$ We also found some old papers, describing single patients with PAN and renal vein thrombosis. ${ }^{15,16}$ Possible explanation is that obliteration of the arterioles that supply the walls of the veins may be causative for the venous thrombosis.

Ureteric stricture, along with orchitis and peri-renal hemorrhage is listed among urogenital complications of PAN.,11. There are several case reports, describing ureteral strictures in PAN, always as unusual presentation of the disease. ${ }^{17-25}$; thus, phlegmonous-ulcerous ureteritis with fibrinous periureteritis found in our patient represent a very rare type of lesion. In our case the pathology findings, demonstrating the specific damage of renal artery, intra-renal vasculature, renal vein and ureter, were confirmative for PAN. With those findings our patient had altogether 7 out of 10 characteristic features of PAN. No crescents 
or any other kind of glomerular lesions were found, which, along with negative ANCA, completely ruled out the diagnosis of ANCAassociated vascultis.

Treatment options for PAN include corticosteroids and immunosuppressant's. Clinical trials suggest that corticosteroids alone are adequate first line therapy for patients with PAN with a FFS of zero however $40 \%$ of them may require additional immunosuppression. Patients with a FFS $\geq 1$ should receive intermittent intravenous cyclophosphamide in addition to corticosteroids. ${ }^{26,27}$ In the steroidrefractory and recurrent PAN rituximab and infliximab or plasma exchanges has been used occasionally. ${ }^{28-30}$ As our patient had FFS 2 (proteinuria and elevated serum creatinine), after nephrectomy, which was indicated given the combination of renal artery aneurism and stenosis with peri-vasal hematoma, kidney shrinking and absence of kidney function, he was started on the combination of prednisone and cyclophosphamide. Almost 2months after nephrectomy and 6 weeks under the immunosuppression all his symptoms resolved, and he is continued on the immunosuppression with intermittent i.v. cyclophosphamide and slow tapering of the oral prednisone dosage. We conclude that this case represent a rare combination of PAN manifestations, demanding multidisciplinary approach. Indeed, he had renal artery aneurism and occlusion with peri-vasal hematoma, multiple kidney infarctions, renal vein thrombosis, necrotizing ureteritis with periureteritis, and orchitis, and a team including radiologist, vascular surgeon, urologist, nephrologist and nephropathologist participated in the diagnostics and treatment.

\section{Acknowledgments}

Natalya Ivanova, Igor Lukyanov, Oksana Paklyna and Elena Zvonova.

\section{Conflicts of interest}

All authors declared there are no conflicts of interest.

\section{Funding}

None.

\section{References}

1. Jennette JC, Falk RJ, Bacon PA, et al. Revised International Chapel Hill Consensus Conference Nomenclature of Vasculitides. Arthritis Rheum. 2013;65(1):1-11

2. Forbess L, Bannykh S. Polyarteritis nodosa. Rheum Dis Clin North Am. 2015;41(1):33-46.

3. Lightfoot RW, Michel BA, Bloch DA, et al. The American College of Rheumatology 1990 criteria for the classification of polyarteritis nodosa. Arthritis Rheum. 1990;33(8):1088-1093.

4. Stone JH. Polyarteritis nodosa. JAMA. 2002;288(13):1632-1639.

5. Colmegna I, Maldonado-Cocco JA. Polyarteritis nodosa revisited. Curr Rheumatol Rep. 2005;7(4):288-296.

6. Guillevin L, Lhote F, Cohen P, et al. Polyarteritis nodosa related to hepatitis $\mathrm{b}$ virus a prospective study with long-term observation of 41 patients. Medicine. 1995;74(5):238-253.

7. Mahr A, Guillevin L, Poissonnet M, et al. Prevalences of polyarteritis nodosa, microscopic polyangiitis, Wegener's granulomatosis, and Churg-Strauss syndrome in a French urban multiethnic population in 2000: a capture-recapture estimate. Arthritis Rheum. 2004;51(1):92-99.

8. Guillevin L, Lhote F, Gayraud M, et al. Prognostic factors in polyarteritis nodosa and Churg-Strauss syndrome. A prospective study in 342 patients. Medicine (Baltimore). 1996;75(1):17-28.
9. Agarwal A, Bansal M, Pandey R, et al. Bilateral subcapsular and perinephric hemorrhage as the initial presentation of polyarteritis nodosa. Intern Med. 2012;51(9):1073-1076.

10. Zhang JQ, Fielding JR, Zou KH. Etiology of spontaneous perirenal hemorrhage:a meta-analysis. J Urol. 2002;167:1593-1596.

11. Peracha J, Morgan MD.Urological manifestations and treatment of the primary systemic vasculitides. World J Clin Urol. 2015;4(1):5-20.

12. Albert DA, Rimon D, Silverstein MD. The diagnosis of polyarteritis nodosa. A literature-based decision analysis approach. Arthritis Rheum. 1998;31(9):1117-1127.

13. Gheita TA, Khairy NA, Nasrallah MM, et al. Subclinical renal involvement in essential cryoglobulinemic vasculitis and classic polyarteritis nodosa. Joint Bone Spine. 2012;79(3):274-280.

14. Azevedo LMO, Hernández SB, Alcobendas JV. Acute kidney failure due to polyarteritis nodosa. Medicina Clínica. 2017;148(2):97-99.

15. Beard MEJ, Taylor DJE. Renal vein thrombosis in cases of polyarteritis nodosa and of the Henoch-Schoenlein syndrome. J Clin Pathol. 1959;22(4):395-400.

16. Laville M, Aguilera D, Maillet PJ, et al. The Prognosis of Renal Vein Thrombosis:A Re-evaluation of 27 Cases. Nephrol Dial Transplant. 1988;3(3):247-256

17. Samellas W, Bellonias E, Papacharalampous N. Polyarteritis nodosa:ureteral involvement. J Urol. 1971;105(2):186-187.

18. Ekeland A, Sander S. Stenosis of the ureter caused by periarteritis. J Oslo City Hosp. 1974;24(6):78-82.

19. Glanz I, Grünebaum M. Ureteral changes in polyarteritis nodosa as seen during excretory urography. J Urol. 1976;116(6):731-733.

20. Abós Fanlo P, Vilardell Tarrés M, Pastor Mouron J, et al. Secondary hydronephrosis to polyarteritis nodosa. Eur Urol. 1979;5(3):211-213.

21. Melin JP, Lemaire P, Birembaut P, , et al. Polyarteritis nodosa with bilateral ureteric involvement. Nephron. 1982;32(1):87-89.

22. Duclos JM, Bensadoun H, Herreman G, et al. Pseudotumor of the ureter manifesting periarteritis nodosa. Ann Urol (Paris). 1984;18:418-419.

23. Hefty TR, Bonafede P, Stenzel P. Bilateral ureteral stricture from polyarteritis nodosa. J Urol. 1989;141(3):600-601.

24. Kaskarelis IS, Zarifi M, Dantis P, et al. Bilateral ureteral involvement in polyarteritis nodosa. Scand J Urol Nephrol. 2010;29(3):323-326.

25. Bulbuloglu E, Kantarceken B, Yuksel M, et al. An unusual presentation of polyarteritis nodosa:A case report. West Indian Med J. 2006;55(1):5659.

26. Guillevin L, Cohen P, Mahr A, et al. Treatment of polyarteritis nodosa and microscopic polyangiitis with poor prognosis factors:a prospective trial comparing glucocorticoids and six or twelve cyclophosphamide pulses in sixty-five patients. Arthritis Rheum. 2003;49(1):93-100.

27. Ribi C, Cohen P, Pagnoux C, et al. Treatment of polyarteritis nodosa and microscopic polyangiitis without poor-prognosis factors:A prospective randomized study of one hundred twenty-four patients. Arthritis Rheum. 2010;62:1186-1197.

28. Seri Y, Shoda $\mathrm{H}$, Hanata N, et al. A case of refractory polyarteritis nodosa successfully treated with rituximab. Mod Rheumatol. 2015;12:1-3.

29. Campanilho-Marques R, Ramos F, Canhão $H$, et al. Remission induced by infliximab in a childhood polyarteritis nodosa refractory to conventional immunosuppression and rituximab. Joint Bone Spine. 2014;81(3):277-278.

30. de Luna G, Chauveau D, Aniort J, et al. Plasma exchanges for the treatment of severe systemic necrotizing vasculitides in clinical daily practice: Data from the French Vasculitis Study Group. J Autoimmun. 2015;65:49-55. 